depression. Further studies with the same methodology that examines and separates central and peripheral olfactory processing are needed. Another review showed robust olfactory deficits in schizophrenia and at-risk youths, what indicates that olfactory measures may be a useful marker of schizophrenia risk status. Finally, a systematic review compared olfactory function in FTD, depression, schizophrenia and bipolar disorder. Results revealed that odor identification but not discrimination was severely impaired in FTD, both were impaired in schizophrenia, while no olfactory impairments were observed in depression. Findings in bipolar disorder were mixed. This review showed that testing odor identification and discrimination differentiates FTD from depression and schizophrenia, but not from bipolar disorder. It is possible to conclude that olfactory dysfunction occurs in schizophrenia and dementia but not in depression.

\title{
540 - COGNITIVE STIMULATION THROUGH OROICOGNITIVE, A VIRTUAL REALITY APP FOR OLDER PEOPLE
}

\section{OBJECTIVE:}

Adriana Gómez, Janeth Carreño, Alvaro Berroa, Ane Balenciaga

To analyze the effectiveness of cognitive stimulation carried out through OroiCognitive, a virtual reality app, in older people, as well as its acceptance and attractiveness to them.

\section{METHOD:}

The research was carried out with 31 participants with a number two or three in the global deterioration scale (GDS).

15 of these participants were part of the control group, and 16 of the experimental group.

The intervention through virtual reality, with exercises more similar to daily activities, allows working on attention, language, memory, orientation, visuospatial skills and executive functions.

It was carried out in 12 sessions, 3 times a week, lasting 25 minutes each one, individually. Bouth groups were evaluated using the MINI-MENTAL Cognitive Examination and some subtest of the Weschler Intelligence Scale for adults (WAIS-IV).

\section{RESULTS:}

Regarding to the effectiveness of the stimulation, the results show significant improvements in vocabulary and information in those with GDS2, in the experimental group.

No statistically significant improvements were found in the rest of the areas. Regarding the assessment of the tool, $69 \%$ of the participants rated it as quite useful and interesting. According to the qualitative data collected by the therapists, the users were happier and more animated during and after the intervention.

\section{1 - MRI-BASED MEASUREMENT OF HIPPOCAMPAL VOLUME IN PATIENTS WITH DEMENTIA}

Dr. Supriya Satapathy, M.D, Consultant Psychiatrist, Brain Mind Behavior Neurosciences Research Institute, Visakhapatnam, Andhra Pradesh, India.

Dr. D. Phani Bhushan, M.D, D.P.M., Professor \& Head, Department of Psychiatry

Dr. T. Nageshwar Rao, M.D., Professor, Department of Radio-diagnosis

Dr. M. Satyanarayana, M.D., D.M., Assistant Professor, Interventional Neuroradiologist

\section{Background:}

- Dementia due to probable Alzheimer's disease (AD) represents between 60 and $80 \%$ of all dementias. The total number of estimated AD cases worldwide by 2030 is 65.7 million and 115.4 million by 2050; this represents a twofold population increase in the next 20 years.

- Magnetic resonance imaging (MRI) has been the primary tool of interest to link hippocampal volume loss with dementia firmly. 